\title{
Optimal Control Problems for Nonlinear Variational Evolution Inequalities
}

\author{
Eun-Young Ju and Jin-Mun Jeong \\ Department of Applied Mathematics, Pukyong National University, Busan 608-737, Republic of Korea \\ Correspondence should be addressed to Jin-Mun Jeong; jmjeong@pknu.ac.kr
}

Received 12 November 2012; Revised 4 January 2013; Accepted 6 January 2013

Academic Editor: Ryan Loxton

Copyright (C) 2013 E.-Y. Ju and J.-M. Jeong. This is an open access article distributed under the Creative Commons Attribution License, which permits unrestricted use, distribution, and reproduction in any medium, provided the original work is properly cited.

We deal with optimal control problems governed by semilinear parabolic type equations and in particular described by variational inequalities. We will also characterize the optimal controls by giving necessary conditions for optimality by proving the Gâteaux differentiability of solution mapping on control variables.

\section{Introduction}

In this paper, we deal with optimal control problems governed by the following variational inequality in a Hilbert space $H$ :

$$
\begin{aligned}
& \left(x^{\prime}(t)+A x(t), x(t)-z\right)+\phi(x(t))-\phi(z) \\
& \leq(f(t, x(t))+B u(t), x(t)-z), \\
& \text { a.e., } 0<t \leq T, z \in V, \\
& x(0)=x_{0} .
\end{aligned}
$$

Here, $A$ is a continuous linear operator from $V$ into $V^{*}$ which is assumed to satisfy Gårding's inequality, where $V$ is dense subspace in $H$. Let $\phi: V \rightarrow(-\infty,+\infty]$ be a lower semicontinuous, proper convex function. Let $\mathcal{U}$ be a Hilbert space of control variables, and let $B$ be a bounded linear operator from $\mathcal{U}$ into $L^{2}(0, T ; H)$. Let $\mathscr{U}_{\text {ad }}$ be a closed convex subset of $\mathcal{U}$, which is called the admissible set. Let $J=J(v)$ be a given quadratic cost function (see (61) or (103)). Then we will find an element $u \in \mathscr{U}_{\text {ad }}$ which attains minimum of $J(v)$ over $\mathscr{U}_{\text {ad }}$ subject to (1).

Recently, initial and boundary value problems for permanent magnet technologies have been introduced via variational inequalities in $[1,2]$ and nonlinear variational inequalities of semilinear parabolic type in $[3,4]$. The papers treating the variational inequalities with nonlinear perturbations are not many. First of all, we deal with the existence and a variation of constant formula for solutions of the nonlinear functional differential equation (1) governed by the variational inequality in Hilbert spaces in Section 2.

Based on the regularity results for solution of (1), we intend to establish the optimal control problem for the cost problems in Section 3. For the optimal control problem of systems governed by variational inequalities, see $[1,5]$. We refer to $[6,7]$ to see the applications of nonlinear variational inequalities. Necessary conditions for state constraint optimal control problems governed by semilinear elliptic problems have been obtained by Bonnans and Tiba [8] using methods of convex analysis (see also [9]).

Let $x_{u}$ stand for solution of (1) associated with the control $u \in \mathcal{U}$. When the nonlinear mapping $f$ is Lipschitz continuous from $\mathbb{R} \times V$ into $H$, we will obtain the regularity for solutions of (1) and the norm estimate of a solution of the above nonlinear equation on desired solution space. Consequently, in view of the monotonicity of $\partial \phi$, we show that the mapping $u \mapsto x_{u}$ is continuous in order to establish the necessary conditions of optimality of optimal controls for various observation cases.

In Section 4, we will characterize the optimal controls by giving necessary conditions for optimality. For this, it is necessary to write down the necessary optimal condition due to the theory of Lions [9]. The most important objective of such a treatment is to derive necessary optimality conditions 
that are able to give complete information on the optimal control.

Since the optimal control problems governed by nonlinear equations are nonsmooth and nonconvex, the standard methods of deriving necessary conditions of optimality are inapplicable here. So we approximate the given problem by a family of smooth optimization problems and afterwards tend to consider the limit in the corresponding optimal control problems. An attractive feature of this approach is that it allows the treatment of optimal control problems governed by a large class of nonlinear systems with general cost criteria.

\section{Regularity for Solutions}

If $H$ is identified with its dual space we may write $V \subset H \subset V^{*}$ densely and the corresponding injections are continuous. The norm on $V, H$, and $V^{*}$ will be denoted by $\|\cdot\|,|\cdot|$, and $\|\cdot\|_{*}$, respectively. The duality pairing between the element $v_{1}$ of $V^{*}$ and the element $v_{2}$ of $V$ is denoted by $\left(v_{1}, v_{2}\right)$, which is the ordinary inner product in $H$ if $v_{1}, v_{2} \in H$.

For $l \in V^{*}$ we denote $(l, v)$ by the value $l(v)$ of $l$ at $v \in V$. The norm of $l$ as element of $V^{*}$ is given by

$$
\|l\|_{*}=\sup _{v \in V} \frac{|(l, v)|}{\|v\|} .
$$

Therefore, we assume that $V$ has a stronger topology than $H$ and, for brevity, we may regard that

$$
\|u\|_{*} \leq|u| \leq\|u\|, \quad \forall u \in V .
$$

Let $a(\cdot, \cdot)$ be a bounded sesquilinear form defined in $V \times V$ and satisfying Gårding’s inequality

$$
\operatorname{Re} a(u, u) \geq \omega_{1}\|u\|^{2}-\omega_{2}|u|^{2},
$$

where $\omega_{1}>0$ and $\omega_{2}$ is a real number. Let $A$ be the operator associated with this sesquilinear form:

$$
(A u, v)=a(u, v), \quad u, v \in V .
$$

Then $-A$ is a bounded linear operator from $V$ to $V^{*}$ by the Lax-Milgram Theorem. The realization of $A$ in $H$ which is the restriction of $A$ to

$$
D(A)=\{u \in V: A u \in H\}
$$

is also denoted by $A$. From the following inequalities

$$
\begin{aligned}
\omega_{1}\|u\|^{2} & \leq \operatorname{Re} a(u, u)+\omega_{2}|u|^{2} \leq C|A u||u|+\omega_{2}|u|^{2} \\
& \leq\left(C|A u|+\omega_{2}|u|\right)|u| \leq \max \left\{C, \omega_{2}\right\}\|u\|_{D(A)}|u|
\end{aligned}
$$

where

$$
\|u\|_{D(A)}=\left(|A u|^{2}+|u|^{2}\right)^{1 / 2}
$$

is the graph norm of $D(A)$, it follows that there exists a constant $C_{0}>0$ such that

$$
\|u\| \leq C_{0}\|u\|_{D(A)}^{1 / 2}|u|^{1 / 2}
$$

Thus we have the following sequence

$$
D(A) \subset V \subset H \subset V^{*} \subset D(A)^{*},
$$

where each space is dense in the next one with continuous injection.

Lemma 1. With the notations (9) and (10), we have

$$
\begin{gathered}
\left(V, V^{*}\right)_{1 / 2,2}=H, \\
(D(A), H)_{1 / 2,2}=V,
\end{gathered}
$$

where $\left(V, V^{*}\right)_{1 / 2,2}$ denotes the real interpolation space between $V$ and $V^{*}$ (Section 1.3 .3 of [10]).

It is also well known that $A$ generates an analytic semigroup $S(t)$ in both $H$ and $V^{*}$. For the sake of simplicity we assume that $\omega_{2}=0$ and hence the closed half plane $\{\lambda$ : $\operatorname{Re} \lambda \geq 0\}$ is contained in the resolvent set of $A$.

If $X$ is a Banach space, $L^{2}(0, T ; X)$ is the collection of all strongly measurable square integrable functions from $(0, T)$ into $X$ and $W^{1,2}(0, T ; X)$ is the set of all absolutely continuous functions on $[0, T]$ such that their derivative belongs to $L^{2}(0, T ; X) . C([0, T] ; X)$ will denote the set of all continuously functions from $[0, T]$ into $X$ with the supremum norm. If $X$ and $Y$ are two Banach spaces, $\mathscr{L}(X, Y)$ is the collection of all bounded linear operators from $X$ into $Y$, and $\mathscr{L}(X, X)$ is simply written as $\mathscr{L}(X)$. Here, we note that by using interpolation theory we have

$$
L^{2}(0, T ; V) \cap W^{1,2}\left(0, T ; V^{*}\right) \subset C([0, T] ; H) .
$$

First of all, consider the following linear system:

$$
\begin{aligned}
& x^{\prime}(t)+A x(t)=k(t), \\
& x(0)=x_{0} .
\end{aligned}
$$

By virtue of Theorem 3.3 of [11] (or Theorem 3.1 of [12, $13]$ ), we have the following result on the corresponding linear equation of (13).

Lemma 2. Suppose that the assumptions for the principal operator A stated above are satisfied. Then the following properties hold.

(1) For $x_{0} \in V=(D(A), H)_{1 / 2,2}$ (see Lemma 1) and $k \epsilon$ $L^{2}(0, T ; H), T>0$, there exists a unique solution $x$ of (13) belonging to

$$
L^{2}(0, T ; D(A)) \cap W^{1,2}(0, T ; H) \subset C([0, T] ; V)
$$

$$
\text { and satisfying }
$$

$$
\|x\|_{L^{2}(0, T ; D(A)) \cap W^{1,2}(0, T ; H)} \leq C_{1}\left(\left\|x_{0}\right\|+\|k\|_{L^{2}(0, T ; H)}\right),
$$

where $C_{1}$ is a constant depending on $T$.

(2) Let $x_{0} \in H$ and $k \in L^{2}\left(0, T ; V^{*}\right), T>0$. Then there exists a unique solution $x$ of (13) belonging to

$$
L^{2}(0, T ; V) \cap W^{1,2}\left(0, T ; V^{*}\right) \subset C([0, T] ; H)
$$


and satisfying

$$
\|x\|_{L^{2}(0, T ; V) \cap W^{1,2}\left(0, T ; V^{*}\right)} \leq C_{1}\left(\left|x_{0}\right|+\|k\|_{L^{2}\left(0, T ; V^{*}\right)}\right),
$$

where $C_{1}$ is a constant depending on $T$.

Let $f$ be a nonlinear single valued mapping from $[0, \infty) \times$ $V$ into $H$.

(F) We assume that

$$
\left|f\left(t, x_{1}\right)-f\left(t, x_{2}\right)\right| \leq L\left\|x_{1}-x_{2}\right\|,
$$

for every $x_{1}, x_{2} \in V$.

Let $Y$ be another Hilbert space of control variables and take $\mathcal{U}=L^{2}(0, T ; Y)$ as stated in the Introduction. Choose a bounded subset $U$ of $Y$ and call it a control set. Let us define an admissible control $\mathcal{U}_{\text {ad }}$ as

$\mathcal{U}_{\mathrm{ad}}=\left\{u \in L^{2}(0, T ; Y): u\right.$ is strongly measurable function satisfying $u(\mathrm{t}) \in U$ for almost all $t\}$.

Noting that the subdifferential operator $\partial \phi$ is defined by

$$
\partial \phi(x)=\left\{x^{*} \in V^{*} ; \phi(x) \leq \phi(y)+\left(x^{*}, x-y\right), y \in V\right\},
$$

the problem (1) is represented by the following nonlinear functional differential problem on $H$ :

$$
\begin{array}{r}
x^{\prime}(t)+A x(t)+\partial \phi(x(t)) \ni f(t, x(t))+B u(t), \\
0<t \leq T,
\end{array}
$$

$x(0)=x_{0}$.

Referring to Theorem 3.1 of [3], we establish the following results on the solvability of (1).

Proposition 3. (1) Let the assumption $(F)$ be satisfied. Assume that $u \in L^{2}(0, T ; Y), B \in \mathscr{L}\left(Y, V^{*}\right)$, and $x_{0} \in \overline{D(\phi)}$ where $\overline{D(\phi)}$ is the closure in $H$ of the set $D(\phi)=\{u \in V: \phi(u)<\infty\}$. Then, (1) has a unique solution

$$
x \in L^{2}(0, T ; V) \cap C([0, T] ; H)
$$

which satisfies

$$
x^{\prime}(t)=B u(t)-A x(t)-(\partial \phi)^{0}(x(t))+f(t, x(t)),
$$

where $(\partial \phi)^{0}: H \rightarrow H$ is the minimum element of $\partial \phi$ and there exists a constant $C_{2}$ depending on $T$ such that

$$
\|x\|_{L^{2} \cap C} \leq C_{2}\left(1+\left|x_{0}\right|+\|B u\|_{L^{2}\left(0, T ; V^{*}\right)}\right),
$$

where $C_{2}$ is some positive constant and $L^{2} \cap C=L^{2}(0, T ; V) \cap$ $C([0, T] ; H)$.

Furthermore, if $B \in \mathscr{L}(Y, H)$ then the solution $x$ belongs to $W^{1,2}(0, T ; H)$ and satisfies

$$
\|x\|_{W^{1,2}(0, T ; H)} \leq C_{2}\left(1+\left|x_{0}\right|+\|B u\|_{L^{2}(0, T ; H)}\right) .
$$

(2) We assume the following.

(A) $A$ is symmetric and there exists $h \in H$ such that for every $\epsilon>0$ and any $y \in D(\phi)$

$$
\begin{gathered}
J_{\epsilon}(y+\epsilon h) \in D(\phi), \quad \phi\left(J_{\epsilon}(y+\epsilon h)\right) \leq \phi(y), \\
\text { where } J_{\epsilon}=(I+\epsilon A)^{-1} .
\end{gathered}
$$

Then for $u \in L^{2}(0, T ; Y), B \in \mathscr{L}(Y, H)$, and $x_{0} \in \overline{D(\phi)} \cap V$ (1) has a unique solution

$$
x \in L^{2}(0, T ; D(A)) \cap W^{1,2}(0, T ; H) \cap C([0, T] ; H),
$$

which satisfies

$$
\|x\|_{L^{2} \cap W^{1,2} \cap C} \leq C_{2}\left(1+\left\|x_{0}\right\|+\|B u\|_{L^{2}(0, T ; H)}\right) .
$$

Remark 4. In terms of Lemma 1, the following inclusion

$$
L^{2}(0, T ; V) \cap W^{1,2}\left(0, T ; V^{*}\right) \subset C([0, T] ; H)
$$

is well known as seen in (9) and is an easy consequence of the definition of real interpolation spaces by the trace method (see $[4,13])$.

The following Lemma is from Brézis [14, Lemma A.5].

Lemma 5. Let $m \in L^{1}(0, T ; \mathbb{R})$ satisfying $m(t) \geq 0$ for all $t \in$ $(0, T)$ and $a \geq 0$ be a constant. Let $b$ be a continuous function on $[0, T] \subset \mathbb{R}$ satisfying the following inequality:

$$
\frac{1}{2} b^{2}(t) \leq \frac{1}{2} a^{2}+\int_{0}^{t} m(s) b(s) d s, \quad t \in[0, T] .
$$

Then,

$$
|b(t)| \leq a+\int_{0}^{t} m(s) d s, \quad t \in[0, T] .
$$

For each $\left(x_{0}, u\right) \in H \times L^{2}(0, T ; Y)$, we can define the continuous solution mapping $\left(x_{0}, u\right) \mapsto x$. Now, we can state the following theorem.

Theorem 6. (1) Let the assumption (F) be satisfied, $x_{0} \in H$, and $B \in \mathscr{L}\left(Y, V^{*}\right)$. Then the solution $x$ of (1) belongs to $x \in$ $L^{2}(0, T ; V) \cap C([0, T] ; H)$ and the mapping

$$
\begin{aligned}
H \times L^{2}(0, T ; Y) \ni\left(x_{0}, u\right) \\
\quad \longmapsto x \in L^{2}(0, T ; V) \cap C([0, T] ; H)
\end{aligned}
$$

is Lipschtz continuous; that is, suppose that $\left(x_{0 i}, u_{i}\right) \in H \times$ $L^{2}(0, T ; Y)$ and $x_{i}$ be the solution of $(1)$ with $\left(x_{0 i}, u_{i}\right)$ in place of $\left(x_{0}, u\right)$ for $i=1,2$,

$$
\begin{aligned}
& \left\|x_{1}-x_{2}\right\|_{L^{2}(0, T ; V) \cap C([0, T] ; H)} \\
& \quad \leq C\left\{\left|x_{01}-x_{02}\right|+\left\|u_{1}-u_{2}\right\|_{L^{2}(0, T ; Y)}\right\},
\end{aligned}
$$

where $C$ is a constant. 
(2) Let the assumptions $(A)$ and $(F)$ be satisfied and let $B \in \mathscr{L}(Y, H)$ and $x_{0} \in \overline{D(\phi)} \cap V$. Then $x \in L^{2}(0, T ; D(A)) \cap$ $W^{1,2}(0, T ; H)$, and the mapping

$$
\begin{aligned}
V \times L^{2}(0, T ; Y) \ni\left(x_{0}, u\right) \\
\quad \longmapsto x \in L^{2}(0, T ; D(A)) \cap W^{1,2}(0, T ; H)
\end{aligned}
$$

is continuous.

Proof. (1) Due to Proposition 3, we can infer that (1) possesses a unique solution $x \in L^{2}(0, T ; V) \cap C([0, T] ; H)$ with the data condition $\left(x_{0}, u\right) \in H \times L^{2}(0, T ; Y)$. Now, we will prove the inequality (33). For that purpose, we denote $x_{1}-x_{2}$ by $X$. Then

$$
\begin{aligned}
& X^{\prime}(t)+A X(t)+\partial \phi\left(x_{1}(t)\right)-\partial \phi\left(x_{2}(t)\right) \\
& \quad \ni f\left(t, x_{1}(t)\right)-f\left(t, x_{2}(t)\right) \\
& \quad+B\left(u_{1}(t)-u_{2}(t)\right), \quad 0<t \leq T, \\
& X(0)=x_{01}-x_{02} .
\end{aligned}
$$

Multiplying on the above equation by $X(t)$, we have

$$
\begin{aligned}
& \frac{1}{2} \frac{d}{d t}|X(t)|^{2}+\omega_{1}\|X(t)\|^{2} \\
& \leq \omega_{2}|X(t)|^{2} \\
& \quad+\left\{\left|f\left(t, x_{1}(t)\right)-f\left(t, x_{2}(t)\right)\right|+\left|B\left(u_{1}(t)-u_{2}(t)\right)\right|\right\} \\
& \quad \times|X(t)| .
\end{aligned}
$$

Put

$$
H(t)=\left(L\|X(t)\|+\left|B\left(u_{1}(t)-u_{2}(t)\right)\right|\right)|X(t)| .
$$

By integrating the above inequality over $[0, t]$, we have

$$
\begin{aligned}
& \frac{1}{2}|X(t)|^{2}+\omega_{1} \int_{0}^{t}\|X(s)\|^{2} d s \\
& \quad \leq \frac{1}{2}\left|x_{01}-x_{02}\right|^{2}+\omega_{2} \int_{0}^{t}|X(s)|^{2} d s+\int_{0}^{t} H(s) d s .
\end{aligned}
$$

Note that

$$
\begin{aligned}
\frac{d}{d t}\left\{e^{-2 \omega_{2} t} \int_{0}^{t}|X(s)|^{2} d s\right\} \\
\leq 2 e^{-2 \omega_{2} t}\left\{\frac{1}{2}|X(t)|^{2}-\omega_{2} \int_{0}^{t}|X(s)|^{2} d s\right\} \\
\leq 2 e^{-2 \omega_{2} t}\left\{\frac{1}{2}\left|x_{01}-x_{02}\right|^{2}+\int_{0}^{t} H(s) d s\right\},
\end{aligned}
$$

integrating the above inequality over $(0, t)$, we have

$$
\begin{aligned}
e^{-2 \omega_{2} t} \int_{0}^{t}|X(s)|^{2} d s & \leq 2 \int_{0}^{t} e^{-2 \omega_{2} \tau}\left\{\frac{1}{2}\left|x_{01}-x_{02}\right|^{2}+\int_{0}^{\tau} H(s) d s\right\} d \tau \\
= & \frac{1-e^{-2 \omega_{2} t}}{2 \omega_{2}}\left|x_{01}-x_{02}\right|^{2}+2 \int_{0}^{t} \int_{s}^{t} e^{-2 \omega_{2} \tau} d \tau H(s) d s \\
= & \frac{1-e^{-2 \omega_{2} t}}{2 \omega_{2}}\left|x_{01}-x_{02}\right|^{2} \\
& +\frac{1}{\omega_{2}} \int_{0}^{t}\left(e^{-2 \omega_{2} s}-e^{-2 \omega_{2} t}\right) H(s) d s .
\end{aligned}
$$

Thus, we get

$$
\begin{aligned}
& \omega_{2} \int_{0}^{t}|X(s)|^{2} d s \leq \frac{1}{2}\left(e^{2 \omega_{2} t}-1\right)\left|x_{01}-x_{02}\right|^{2} \\
& +\int_{0}^{t}\left(e^{2 \omega_{2}(t-s)}-1\right) H(s) d s .
\end{aligned}
$$

Combining this with (38) it holds that

$$
\begin{aligned}
\frac{1}{2}|X(t)|^{2} & +\omega_{1} \int_{0}^{t}\|X(s)\|^{2} d s \leq \frac{1}{2} e^{2 \omega_{2} t}\left|x_{01}-x_{02}\right|^{2} \\
& +\int_{0}^{t} e^{2 \omega_{2}(t-s)} H(s) d s .
\end{aligned}
$$

By Lemma 5, the following inequality

$$
\begin{aligned}
& \frac{1}{2}\left(e^{-\omega_{2} t}|X(t)|\right)^{2}+\omega_{1} e^{-2 \omega_{2} t} \int_{0}^{t}\|X(s)\|^{2} d s \\
& \leq \frac{1}{2}\left|x_{01}-x_{02}\right|^{2} \\
& \quad+\int_{0}^{t} e^{-\omega_{2} s}\left(L\|X(s)\|+\left|B\left(u_{1}(s)-u_{2}(s)\right)\right|\right) e^{-\omega_{2} s}|X(s)| d s
\end{aligned}
$$

implies that

$$
\begin{aligned}
& e^{-\omega_{2} t}|X(t)| \leq\left|x_{01}-x_{02}\right| \\
& \quad+\int_{0}^{t} e^{-\omega_{2} s}\left(L\|X(s)\|+\left|B\left(u_{1}(s)-u_{2}(s)\right)\right|\right) d s .
\end{aligned}
$$


From (42) and (44) it follows that

$$
\begin{aligned}
& \frac{1}{2}|X(t)|^{2}+\omega_{1} \int_{0}^{t}\|X(s)\|^{2} d s \leq \frac{1}{2} e^{2 \omega_{2} t}\left|x_{01}-x_{02}\right|^{2} \\
& \quad+\int_{0}^{t} e^{2 \omega_{2}(t-s)}\left(L\|X(s)\|+\left|B\left(u_{1}(s)-u_{2}(s)\right)\right|\right) e^{\omega_{2} s} \\
& \quad \times\left|x_{01}-x_{02}\right| d s \\
& \quad+\int_{0}^{t} e^{2 \omega_{2}(t-s)}\left(L\|X(s)\|+\left|B\left(u_{1}(s)-u_{2}(s)\right)\right|\right) \\
& \quad \times \int_{0}^{s} e^{\omega_{2}(s-\tau)}\left(L\|X(\tau)\|+\left|B\left(u_{1}(\tau)-u_{2}(\tau)\right)\right|\right) d \tau d s \\
& =I+I I+I I I .
\end{aligned}
$$

Putting

$$
G(s)=\|X(s)\|+\left|B\left(u_{1}(s)-u_{2}(s)\right)\right| .
$$

The third term of the right hand side of (45) is estimated as

$$
\begin{aligned}
I I I & =L^{2} e^{2 \omega_{2} t} \int_{0}^{t} e^{-\omega_{2} s}\|G(s)\| \int_{0}^{s} e^{-\omega_{2} \tau}\|G(\tau)\| d \tau d s \\
& =L^{2} e^{2 \omega_{2} t} \int_{0}^{t} \frac{1}{2} \frac{d}{d s}\left\{\int_{0}^{s} e^{-\omega_{2} \tau}\|G(\tau)\| d \tau\right\}^{2} d s \\
& =\frac{1}{2} L^{2} e^{2 \omega_{2} t}\left\{\int_{0}^{t} e^{-\omega_{2} \tau}\|G(\tau)\| d \tau\right\}^{2} \\
& \leq \frac{1}{2} L^{2} e^{2 \omega_{2} t} \frac{1-e^{-2 \omega_{2} t}}{2 \omega_{2}} \int_{0}^{t}\|G(\tau)\|^{2} d \tau \\
& =\frac{L^{2}}{4 \omega_{2}}\left(e^{2 \omega_{2} t}-1\right) \int_{0}^{t}\|G(s)\|^{2} d s \\
& \leq \frac{L^{2}\left(e^{2 \omega_{2} t}-1\right)}{2 \omega_{2}} \int_{0}^{t}\left(\|X(s)\|^{2}+\left|B\left(u_{1}(s)-u_{2}(s)\right)\right|^{2}\right) d s .
\end{aligned}
$$

The second term of the right hand side of (45) is estimated as

$$
\begin{aligned}
I I= & e^{2 \omega_{2} t} \int_{0}^{t} e^{-\omega_{2} s}\left(L\|X(s)\|+\left|B\left(u_{1}(s)-u_{2}(s)\right)\right|\right) d s \\
& \times\left|x_{01}-x_{02}\right| \\
\leq & \frac{1}{2} e^{2 \omega_{2} t} L^{2} \int_{0}^{t}\left(\|X(s)\|^{2}+\left|B\left(u_{1}(s)-u_{2}(s)\right)\right|^{2}\right) d s \\
& +\frac{1}{2} e^{2 \omega_{2} t}\left|x_{01}-x_{02}\right|^{2} .
\end{aligned}
$$

Thus, from (47) and (48), we apply Gronwall's inequality to (15), and we arrive at

$$
\begin{aligned}
& \frac{1}{2}|X(t)|^{2}+\omega_{1} \int_{0}^{t}\|X(s)\|^{2} d s \\
& \quad \leq C\left(\left|x_{01}-x_{02}\right|^{2}+\int_{0}^{T_{1}}\left|B\left(u_{1}(s)-u_{2}(s)\right)\right|^{2} d s\right),
\end{aligned}
$$

where $C>0$ is a constant. Suppose $\left(x_{0 n}, u_{n}\right) \rightarrow\left(x_{0}, u\right)$ in $H \times$ $L^{2}(0, T ; Y)$, and let $x_{n}$ and $x$ be the solutions (1) with $\left(x_{0 n}, u_{n}\right)$ and $\left(x_{0}, u\right)$, respectively. Then, by virtue of (49), we see that $x_{n} \rightarrow x$ in $L^{2}(0, T, V) \cap C([0, T] ; H)$.

(2) It is easy to show that if $x_{0} \in V$ and $B \in \mathscr{L}(Y, H)$, then $x$ belongs to $L^{2}(0, T ; D(A)) \cap W^{1,2}(0, T ; H)$. Let $\left(x_{0 i}, u_{i}\right) \in$ $V \times L^{2}(0, T ; H)$, and $x_{i}$ be the solution of (1) with $\left(x_{0 i}, u_{i}\right)$ in place of $\left(x_{0}, u\right)$ for $i=1,2$. Then in view of Lemma 2 and assumption $(\mathrm{F})$, we have

$$
\begin{gathered}
\left\|x_{1}-x_{2}\right\|_{L^{2}(0, T ; D(A)) \cap W^{1,2}(0, T ; H)} \\
\leq C_{1}\left\{\left\|x_{01}-x_{02}\right\|+\left\|f\left(\cdot, x_{1}\right)-f\left(\cdot, x_{2}\right)\right\|_{L^{2}(0, T ; H)}\right. \\
\left.+\left\|B\left(u_{1}-u_{2}\right)\right\|_{L^{2}(0, T ; H)}\right\} \\
\leq C_{1}\left\{\left\|x_{01}-x_{02}\right\|+\left\|B\left(u_{1}-u_{2}\right)\right\|_{L^{2}(0, T ; H)}\right. \\
\left.+L\left\|x_{1}-x_{2}\right\|_{L^{2}(0, T: V)}\right\} .
\end{gathered}
$$

Since

$$
x_{1}(t)-x_{2}(t)=x_{01}-x_{02}+\int_{0}^{t}\left(\dot{x}_{1}(s)-\dot{x}_{2}(s)\right) d s
$$

we get, noting that $|\cdot| \leq\|\cdot\|$,

$$
\begin{gathered}
\left\|x_{1}-x_{2}\right\|_{L^{2}(0, T ; H)} \leq \sqrt{T}\left\|x_{01}-x_{02}\right\| \\
+\frac{T}{\sqrt{2}}\left\|x_{1}-x_{2}\right\|_{W^{1,2}(0, T ; H)} .
\end{gathered}
$$

Hence arguing as in (9) we get

$$
\begin{aligned}
\| x_{1}- & x_{2}\left\|_{L^{2}(0, T ; V)} \leq C_{0}\right\| x_{1}-x_{2}\left\|_{L^{2}(0, T ; D(A))}^{1 / 2}\right\| x_{1}-x_{2} \|_{L^{2}(0, T ; H)}^{1 / 2} \\
\leq & C_{0}\left\|x_{1}-x_{2}\right\|_{L^{2}(0, T ; D(A))}^{1 / 2} \\
& \times\left\{T^{1 / 4}\left\|x_{01}-x_{02}\right\|^{1 / 2}+\left(\frac{T}{\sqrt{2}}\right)^{1 / 2}\left\|x_{1}-x_{2}\right\|_{W^{1,2}(0, T ; H)}^{1 / 2}\right\} \\
\leq & C_{0} T^{1 / 4}\left\|x_{01}-x_{02}\right\|^{1 / 2}\left\|x_{1}-x_{2}\right\|_{L^{2}(0, T ; D(A))}^{1 / 2} \\
& +C_{0}\left(\frac{T}{\sqrt{2}}\right)^{1 / 2}\left\|x_{1}-x_{2}\right\|_{L^{2}(0, T ; D(A)) \cap W^{1,2}(0, T ; H)} \\
\leq & 2^{-7 / 4} C_{0}\left\|x_{01}-x_{02}\right\| \\
& +2 C_{0}\left(\frac{T}{\sqrt{2}}\right)^{1 / 2}\left\|x_{1}-x_{2}\right\|_{L^{2}(0, T ; D(A)) \cap W^{1,2}(0, T ; H)} .
\end{aligned}
$$

Combining (50) and (53) we obtain

$$
\begin{aligned}
& \left\|x_{1}-x_{2}\right\|_{L^{2}(0, T ; D(A)) \cap W^{1,2}(0, T ; H)} \\
& \leq C_{1}\left\{\left\|x_{01}-x_{02}\right\|\right\}+\left\|B u_{1}-B u_{2}\right\|_{L^{2}(0, T ; H)} \\
& \quad+2^{-7 / 4} C_{0} C_{1} L\left\|x_{01}-x_{02}\right\| \\
& \quad+2 C_{0} C_{1}\left(\frac{T}{\sqrt{2}}\right)^{1 / 2} L\left\|x_{1}-x_{2}\right\|_{L^{2}(0, T ; D(A)) \cap W^{1,2}(0, T ; H)} .
\end{aligned}
$$


Suppose that

$$
\left(x_{0 n}, u_{n}\right) \longmapsto\left(x_{0}, u\right) \in V \times L^{2}(0, T ; Y) \text {, }
$$

and let $x_{n}$ and $x$ be the solutions (1) with $\left(x_{0 n}, u_{n}\right)$ and $\left(x_{0}, u\right)$, respectively. Let $0<T_{1} \leq T$ be such that

$$
2 C_{0} C_{1}\left(\frac{T_{1}}{\sqrt{2}}\right)^{1 / 2} L<1 .
$$

Then by virtue of (54) with $T$ replaced by $T_{1}$ we see that

$$
x_{n} \longrightarrow x \in L^{2}\left(0, T_{1} ; D(A)\right) \cap W^{1,2}\left(0, T_{1} ; H\right) .
$$

This implies that $\left(x_{n}\left(T_{1}\right),\left(x_{n}\right)_{T_{1}}\right) \mapsto\left(x\left(T_{1}\right), x_{T_{1}}\right)$ in $V \times$ $L^{2}(0, T ; D(A))$. Hence the same argument shows that $x_{n} \mapsto x$ in

$$
L^{2}\left(T_{1}, \min \left\{2 T_{1}, T\right\} ; D(A)\right) \cap W^{1,2}\left(T_{1}, \min \left\{2 T_{1}, T\right\} ; H\right) .
$$

Repeating this process we conclude that $x_{n} \mapsto x$ in $L^{2}(0, T ; D(A)) \cap W^{1,2}(0, T ; H)$.

\section{Optimal Control Problems}

In this section we study the optimal control problems for the quadratic cost function in the framework of Lions [9]. In what follows we assume that the embedding $D(A) \subset V \subset H$ is compact.

Let $Y$ be another Hilbert space of control variables, and $B$ be a bounded linear operator from $Y$ into $H$; that is,

$$
B \in \mathscr{L}(Y, H),
$$

which is called a controller. By virtue of Theorem 6, we can define uniquely the solution map $u \mapsto x(u)$ of $L^{2}(0, T ; Y)$ into $L^{2}(0, T ; V) \cap C([0, T] ; H)$. We will call the solution $x(u)$ the state of the control system (1).

Let $M$ be a Hilbert space of observation variables. The observation of state is assumed to be given by

$$
z(u)=G x(u), \quad G \in \mathscr{L}\left(C\left(0, T ; V^{*}\right), M\right),
$$

where $G$ is an operator called the observer. The quadratic cost function associated with the control system (1) is given by

$$
\begin{aligned}
J(v)= & \left\|G x(v)-z_{d}\right\|_{M}^{2} \\
& +(R v, v)_{L^{2}(0, T ; Y)} \quad \text { for } v \in L^{2}(0, T ; Y),
\end{aligned}
$$

where $z_{d} \in M$ is a desire value of $x(v)$ and $R \in \mathscr{L}\left(L^{2}(0, T ; Y)\right)$ is symmetric and positive; that is,

$$
(R v, v)_{L^{2}(0, T ; Y)}=(v, R v)_{L^{2}(0, T ; Y)} \geq d\|v\|_{L^{2}(0, T ; Y)}^{2}
$$

for some $d>0$. Let $\mathcal{U}_{\text {ad }}$ be a closed convex subset of $L^{2}(0, T ; Y)$, which is called the admissible set. An element $u \in \mathscr{U}_{\text {ad }}$ which attains minimum of $J(v)$ over $\mathscr{u}_{\text {ad }}$ is called an optimal control for the cost function (61).
Remark 7. The solution space $\mathscr{W}$ of strong solutions of (1) is defined by

$$
\mathscr{W}=L^{2}(0, T ; V) \cap W^{1,2}\left(0, T ; V^{*}\right) \subset C([0, T] ; H)
$$

endowed with the norm

$$
\|\cdot\|_{\mathscr{W}}=\max \left\{\|\cdot\|_{L^{2}(0, T ; V)},\|\cdot\|_{W^{1,2}\left(0, T ; V^{*}\right)}\right\} .
$$

Let $\Omega$ be an open bounded and connected set of $\mathbb{R}^{n}$ with smooth boundary. We consider the observation $G$ of distributive and terminal values (see $[15,16])$.

(1) We take $M=L^{2}((0, T) \times \Omega) \times L^{2}(\Omega)$ and $G \in \mathscr{L}(\mathscr{W}, M)$ and observe

$$
\begin{aligned}
z(v) & =G x(v) \\
& =(x(v ; \cdot), x(v, T)) \in L^{2}((0, T) \times \Omega) \times L^{2}(\Omega) .
\end{aligned}
$$

(2) We take $M=L^{2}((0, T) \times \Omega)$ and $G \in \mathscr{L}(\mathscr{W}, M)$ and observe

$$
z(v)=G x(v)=y^{\prime}(v ; \cdot) \in L^{2}((0, T) \times \Omega) .
$$

The above observations are meaningful in view of the regularity of (1) by Proposition 3.

Theorem 8. (1) Let the assumption (F) be satisfied. Assume that $B \in \mathscr{L}\left(Y, V^{*}\right)$ and $x_{0} \in \overline{D(\phi)}$. Let $x(u)$ be the solution of (1) corresponding to $u$. Then the mapping $u \mapsto x(u)$ is compact from $L^{2}(0, T ; Y)$ to $L^{2}(0, T ; H)$.

(2) Let the assumptions ( $A)$ and $(F)$ be satisfied. If $B \in$ $\mathscr{L}(Y, H)$ and $x_{0} \in \overline{D(\phi)} \cap V$, then the mapping $u \mapsto x(u)$ is compact from $L^{2}(0, T ; Y)$ to $L^{2}(0, T ; V)$.

Proof. (1) We define the solution mapping $S$ from $L^{2}(0, T ; Y)$ to $L^{2}(0, T ; H)$ by

$$
S u=x(u), \quad u \in L^{2}(0, T ; Y) .
$$

In virtue of Lemma 2, we have

$$
\begin{aligned}
& \|S u\|_{L^{2}(0, T ; V) \cap W^{1,2}\left(0, T ; V^{*}\right)} \\
& =\|x(u)\| \leq C_{1}\left\{\left|x_{0}\right|+\|B u\|_{L^{2}\left(0, T ; V^{*}\right)}\right\} .
\end{aligned}
$$

Hence if $u$ is bounded in $L^{2}(0, T ; Y)$, then so is $x(u)$ in $L^{2}(0, T ; V) \cap W^{1,2}\left(0, T ; V^{*}\right)$. Since $V$ is compactly embedded in $H$ by assumption, the embedding $L^{2}(0, T ; V) \cap$ $W^{1,2}\left(0, T ; V^{*}\right) \subset L^{2}(0, T ; H)$ is also compact in view of Theorem 2 of Aubin [17]. Hence, the mapping $u \mapsto S u=x(u)$ is compact from $L^{2}(0, T ; Y)$ to $L^{2}(0, T ; H)$.

(2) If $D(A)$ is compactly embedded in $V$ by assumption, the embedding

$$
L^{2}(0, T ; D(A)) \cap W^{1,2}(0, T ; H) \subset L^{2}(0, T ; V)
$$

is compact. Hence, the proof of (2) is complete.

As indicated in the Introduction we need to show the existence of an optimal control and to give the characterizations of them. The existence of an optimal control $u$ for the cost function (61) can be stated by the following theorem. 
Theorem 9. Let the assumptions $(A)$ and $(F)$ be satisfied and $x_{0} \in \overline{D(\phi)} \cap V$. Then there exists at least one optimal control $u$ for the control problem (1) associated with the cost function (61); that is, there exists $u \in \mathcal{U}_{\text {ad }}$ such that

$$
J(u)=\inf _{v \in \mathscr{U}_{a d}} J(v):=J
$$

Proof. Since $\mathcal{U}_{\text {ad }}$ is nonempty, there is a sequence $\left\{u_{n}\right\} \subset \mathcal{U}_{\text {ad }}$ such that minimizing sequence for the problem (70) satisfies

$$
\inf _{v \in \mathscr{U}_{\mathrm{ad}}} J(v)=\lim _{n \rightarrow \infty} J\left(u_{n}\right)=m
$$

Obviously, $\left\{J\left(u_{n}\right)\right\}$ is bounded. Hence by (62) there is a positive constant $K_{0}$ such that

$$
d\left\|u_{n}\right\|^{2} \leq\left(R u_{n}, u_{n}\right) \leq J\left(u_{n}\right) \leq K_{0} .
$$

This shows that $\left\{u_{n}\right\}$ is bounded in $\mathcal{U}_{\text {ad }}$. So we can extract a subsequence (denoted again by $\left\{u_{n}\right\}$ ) of $\left\{u_{n}\right\}$ and find a $u \in$ $\mathcal{U}_{\text {ad }}$ such that $w-\lim u_{n}=u$ in $U$. Let $x_{n}=x\left(u_{n}\right)$ be the solution of the following equation corresponding to $u_{n}$ :

$$
\begin{aligned}
& x_{n}^{\prime}(t)+A x_{n}(t)+\partial \phi\left(x_{n}(t)\right) \ni f\left(t, x_{n}(t)\right)+B u_{n}(t), \\
& 0<t \leq T, \\
& x_{n}(0)=x_{0} .
\end{aligned}
$$

By (15) and (17) we know that $\left\{x_{n}\right\}$ and $\left\{x_{n}^{\prime}\right\}$ are bounded in $L^{2}(0, T ; V)$ and $L^{2}\left(0, T ; V^{*}\right)$, respectively. Therefore, by the extraction theorem of Rellich's, we can find a subsequence of $\left\{x_{n}\right\}$, say again $\left\{x_{n}\right\}$, and find $x$ such that

$$
\begin{gathered}
x_{n}(\cdot) \longrightarrow x(\cdot) \text { weakly in } L^{2}(0, T ; V) \cap C([0, T] ; H), \\
x_{n}^{\prime} \longrightarrow x^{\prime}, \text { weakly in } L^{2}\left(0, T ; V^{*}\right) .
\end{gathered}
$$

However, by Theorem 8, we know that

$$
x_{n}(\cdot) \longrightarrow x(\cdot), \text { strongly in } L^{2}(0, T ; V) .
$$

From $(\mathrm{F})$ it follows that

$$
f\left(\cdot, x_{n}\right) \longrightarrow f(\cdot, x) \text {, strongly in } L^{2}(0, T ; H) .
$$

By the boundedness of $A$ we have

$$
A x_{n} \longrightarrow A x \text {, strongly in } L^{2}\left(0, T ; V^{*}\right) .
$$

Since $\partial \phi\left(x_{n}\right)$ are uniformly bounded from (73)-(77) it follows that

$$
\begin{array}{r}
\partial \phi\left(x_{n}\right) \longrightarrow f(\cdot, x)+B u-x^{\prime}-A x, \\
\text { weakly in } L^{2}\left(0, T ; V^{*}\right),
\end{array}
$$

and noting that $\partial \phi$ is demiclosed, we have that

$$
f(\cdot, x)+B u-x^{\prime}-A x \in \partial \phi(x) \text { in } L^{2}\left(0, T ; V^{*}\right) .
$$

Thus we have proved that $x(t)$ satisfies a.e. on $(0, T)$ the following equation:

$$
\begin{aligned}
x^{\prime}(t) & +A x(t)+\partial \phi(x(t)) \ni f(t, x(t)) \\
& +B u(t), \quad \text { a.e., } 0<t \leq T, \\
x(0) & =x_{0} .
\end{aligned}
$$

Since $G$ is continuous and $\|\cdot\|_{M}$ is lower semicontinuous, it holds that

$$
\left\|G x(u)-z_{d}\right\|_{M} \leq \liminf _{n \rightarrow \infty}\left\|G x\left(u_{n}\right)-z_{d}\right\|_{M} .
$$

It is also clear from $\lim \inf _{n \rightarrow \infty}\left\|R^{1 / 2} u_{n}\right\|_{L^{2}(0, T ; Y)} \geq$ $\left\|R^{1 / 2} u\right\|_{L^{2}(0, T ; Y)}$ that

$$
\liminf _{n \rightarrow \infty}\left(R u_{n}, u_{n}\right)_{L^{2}(0, T ; Y)} \geq(R u, u)_{L^{2}(0, T ; Y)} .
$$

Thus,

$$
m=\lim _{n \rightarrow \infty} J\left(u_{n}\right) \geq J(u)
$$

But since $J(u) \geq m$ by definition, we conclude $u \in \mathcal{U}_{\text {ad }}$ is a desired optimal control.

\section{Necessary Conditions for Optimality}

In this section we will characterize the optimal controls by giving necessary conditions for optimality. For this it is necessary to write down the necessary optimal condition

$$
D J(u)(v-u) \geq 0, \quad v \in \mathscr{U}_{\mathrm{ad}}
$$

and to analyze (84) in view of the proper adjoint state system, where $D J(u)$ denote the Gâteaux derivative of $J(v)$ at $v=u$. Therefore, we have to prove that the solution mapping $v \mapsto$ $x(v)$ is Gâteaux differentiable at $v=u$. Here we note that from Theorem 6 it follows immediately that

$$
\begin{aligned}
& \lim _{\lambda \rightarrow 0} x(u+\lambda w) \\
& \quad=x(u), \text { strongly in } L^{2}(0, T ; V) \cap C([0, T] ; H) .
\end{aligned}
$$

The solution map $v \mapsto x(v)$ of $L^{2}(0, T ; Y)$ into $L^{2}(0, T ; V) \cap$ $C([0, T] ; H)$ is said to be Gâteaux differentiable at $v=$ $u$ if for any $w \in L^{2}(0, T ; Y)$ there exists a $D x(u) \in$ $\mathscr{L}\left(L^{2}(0, T ; Y), L^{2}(0, T ; V) \cap C([0, T] ; H)\right.$ such that

$$
\left\|\frac{1}{\lambda}(x(u+\lambda w)-x(u))-D x(u) w\right\| \rightarrow 0 \quad \text { as } \lambda \longrightarrow 0 .
$$

The operator $D x(u)$ denotes the Gâteaux derivative of $x(u)$ at $v=u$ and the function $\left.D x(u) w \in L^{2}(0, T ; V) \cap C([0, T] ; H)\right)$ is called the Gâteaux derivative in the direction $w \in L^{2}(0, T ; Y)$, which plays an important part in the nonlinear optimal control problems.

First, as is seen in Corollary 2.2 of Chapter II of [18], let us introduce the regularization of $\phi$ as follows. 
Lemma 10. For every $\epsilon>0$, define

$$
\phi_{\epsilon}(x)=\left\{\frac{\left\|x-J_{\epsilon} x\right\|_{*}^{2}}{2 \epsilon}+\phi\left(J_{\epsilon} x\right): \forall \epsilon>0, x \in H\right\},
$$

where $J_{\epsilon}=(I+\epsilon \phi)^{-1}$. Then the function $\phi_{\epsilon}$ is Fréchet differentiable on $H$ and its Frećhet differential $\partial \phi_{\epsilon}$ is Lipschitz continuous on $H$ with Lipschitz constant $\epsilon^{-1}$. In addition,

$$
\begin{gathered}
\lim _{\epsilon \rightarrow 0} \phi_{\epsilon}(x)=\phi(x), \quad \forall x \in H, \\
\phi\left(J_{\epsilon} x\right) \leq \phi_{\epsilon}(x) \leq \phi(x), \quad \forall \epsilon>0, x \in H, \\
\lim _{\epsilon \rightarrow 0} \partial \phi_{\epsilon}(x)=(\partial \phi)^{0}(x), \quad \forall x \in H,
\end{gathered}
$$

where $(\partial \phi)^{0}(x)$ is the element of minimum norm in the set $\partial \phi(x)$.

Now, we introduce the smoothing system corresponding to (1) as follows.

$$
\begin{aligned}
x^{\prime}(t) & +A x(t)+\partial \phi_{\epsilon}(x(t)) \\
& =f(t, x(t))+B u(t), \quad 0<t \leq T, \\
x(0) & =x_{0} .
\end{aligned}
$$

Lemma 11. Let the assumption $(F)$ be satisfied. Then the solution map $v \mapsto x(v)$ of $L^{2}(0, T ; Y)$ into $L^{2}(0, T ; V) \cap C([0, T] ; H)$ is Lipschtz continuous.

Moreover, let us assume the condition (A) in Proposition 3. Then the map $v \mapsto \partial \phi_{\epsilon}(x(v))$ of $L^{2}(0, T ; Y)$ into $L^{2}(0, T ; H) \cap$ $C\left([0, T] ; V^{*}\right)$ is also Lipschtz continuous.

Proof. We set $w=v-u$. From Theorem 6, it follows immediately that

$$
\|x(u+\lambda w)-x(u)\|_{C([0, T] ; H)} \leq \text { const. }|\lambda|\|w\|_{L^{2}(0, T ; Y)},
$$

so the solution map $v \mapsto x(v)$ of $L^{2}(0, T ; Y)$ into $L^{2}(0, T ; V) \cap$ $C([0, T] ; H)$ is Lipschtz continuous. Moreover, since

$$
\begin{aligned}
\partial \phi_{\epsilon}(x(u ; t))-\partial \phi_{\epsilon}(x(u+\lambda w ; t)) \\
=x^{\prime}(u+\lambda w ; t)-x^{\prime}(u ; t)+A(x(u+\lambda w ; t)-x(u ; t)) \\
-\{f(t, x(u+\lambda w ; t))-f(t, x(u ; t))\}-\lambda B w(t),
\end{aligned}
$$

by the assumption (A) and (2) of Theorem 6, it holds

$$
\begin{aligned}
& \left\|\partial \phi_{\epsilon}(x(u+\lambda w))-\partial \phi_{\epsilon}(x(u))\right\|_{L^{2}(0, T ; H)} \\
& \leq\left\|x^{\prime}(u+\lambda w)-x^{\prime}(u)\right\|_{L^{2}(0, T ; H)} \\
& \quad+\|x(u+\lambda w)-x(u)\|_{L^{2}(0, T ; D(A))} \\
& \quad+L\|x(u+\lambda w)-x(u)\|_{L^{2}(0, T ; V)} \\
& \quad+|\lambda|\|B\|\|w\|_{L^{2}(0, T ; U)} \\
& \leq \text { const. }|\lambda|\|w\|_{L^{2}(0, T ; Y)}
\end{aligned}
$$

and, by the relation (12),

$$
\begin{aligned}
\| \partial \phi_{\epsilon} & (x(u+\lambda w ; t))-\partial \phi_{\epsilon}(x(u ; t)) \|_{*} \\
\leq & \left\|x^{\prime}(u+\lambda w ; t)-x^{\prime}(u ; t)\right\|_{*} \\
& +\|A\|_{\mathscr{L}\left(V, V^{*}\right)}\|(x(u+\lambda w ; t)-x(u ; t))\| \\
& \quad+L\|x(u+\lambda w ; t)-x(u ; t)\|+|\lambda|\|B\||w(t)| \\
\leq & \text { const. }|\lambda|\|w\|_{L^{2}(0, T ; Y)} .
\end{aligned}
$$

So we know that the map $v \mapsto \partial \phi_{\epsilon}(x(v))$ of $L^{2}(0, T ; Y)$ into $L^{2}(0, T ; H) \cap C\left([0, T] ; V^{*}\right)$ is also Lipschtz continuous.

Let the solution space $\mathscr{W}_{1}$ of (1) of strong solutions is defined by

$$
\mathscr{W}_{1}=L^{2}(0, T ; D(A)) \cap W^{1,2}(0, T ; H)
$$

as stated in Remark 7.

In order to obtain the optimality conditions, we require the following assumptions.

(F1) The Gâteaux derivative $\partial_{2} f(t, x)$ in the second argument for $(t, x) \in(0, T) \times V$ is measurable in $t \in(0, T)$ for $x \in V$ and continuous in $x \in V$ for a.e. $t \in(0, T)$, and there exist functions $\theta_{1}, \theta_{2} \in L^{2}\left(\mathbb{R}^{+} ; \mathbb{R}\right)$ such that

$$
\begin{aligned}
\left\|\partial_{2} f(t, x)\right\|_{*} & \leq \theta_{1}(t)+\theta_{2}(\|x\|), \\
& \forall(t, x) \in(0, T) \times V .
\end{aligned}
$$

(F2) The map $x \rightarrow \partial \phi_{\epsilon}(x)$ is Gâteaux differentiable, and the value $D \partial \phi_{\epsilon}(x) D x(u)$ is the Gâteaux derivative of $\partial \phi_{\epsilon}(x) x(u)$ at $u \in L^{2}(0, T ; U)$ such that there exist functions $\theta_{3}, \theta_{4} \in L^{2}\left(\mathbb{R}^{+} ; \mathbb{R}\right)$ such that

$$
\begin{aligned}
& \left\|D \partial \phi_{\epsilon}(x) D x(u)\right\|_{*} \\
& \quad \leq \theta_{3}(t)+\theta_{4}\left(\|u\|_{L^{2}(0, T ; Y)}\right), \quad \forall u \in L^{2}(0, T ; Y) .
\end{aligned}
$$

Theorem 12. Let the assumptions (A), (F1), and (F2) be satisfied. Let $u \in \mathcal{U}_{\mathrm{ad}}$ be an optimal control for the cost function $J$ in (61). Then the following inequality holds:

$$
\begin{aligned}
& \left(C^{*} \Lambda_{M}\left(C x(u)-z_{d}\right), y\right)_{\mathscr{W}_{1}} \\
& \quad+(R u, v-u)_{L^{2}(0, T ; Y)} \geq 0, \quad \forall v \in \mathscr{U}_{\mathrm{ad}}
\end{aligned}
$$

where $y=D x(u)(v-u) \in C\left([0, T] ; V^{*}\right)$ is a unique solution of the following equation:

$$
\begin{aligned}
& y^{\prime}(t)+A y(t)+D(\partial \phi)^{0}(x)(y(t)) \\
& \quad=\partial_{2} f(t, x) y(t)+B w(t), \quad 0<t \leq T, \\
& y(0)=0 .
\end{aligned}
$$

Proof. We set $w=v-u$. Let $\lambda \in(-1,1), \lambda \neq 0$. We set

$$
y=\lim _{\lambda \rightarrow 0} \lambda^{-1}(x(u+\lambda w)-x(u))=D x(u) w .
$$


From (89), we have

$$
\begin{aligned}
x^{\prime} & (u+\lambda w)-x^{\prime}(u)+A(x(u+\lambda w)-x(u)) \\
& +\partial \phi_{\epsilon}(x(u+\lambda w))-\partial \phi_{\epsilon}(x(u)) \\
& =f(\cdot, x(u+\lambda w))-f(\cdot, x(u))+\lambda B w .
\end{aligned}
$$

Then as an immediate consequence of Lemma 11 one obtains

$$
\begin{aligned}
& \lim _{\lambda \rightarrow 0} \frac{1}{\lambda}\left\{\partial \phi_{\epsilon}(x(u+\lambda w ; t))-\partial \phi_{\epsilon}(x(u ; t))\right\}=D \partial \phi_{\epsilon}(x) y(t), \\
& \lim _{\lambda \rightarrow 0} \frac{1}{\lambda}\{f(t, x(u+\lambda w ; t))-f(t, x(u ; t))\}=\partial_{2} f(t, x) y(t),
\end{aligned}
$$

thus, in the sense of (F2), we have that $y=D x(u)(v-u)$ satisfies (98) and the cost $J(v)$ is Gâteaux differentiable at $u$ in the direction $w=v-u$. The optimal condition (84) is rewritten as

$$
\begin{aligned}
(C x & \left.(u)-z_{d}, y\right)_{M}+(R u, v-u)_{L^{2}(0, T ; Y)} \\
= & \left(C^{*} \Lambda_{M}\left(C x(u)-z_{d}\right), y\right)_{\mathscr{W}_{1}} \\
& +(R u, v-u)_{L^{2}(0, T ; Y)} \geq 0, \quad \forall v \in \mathcal{U}_{\mathrm{ad}} .
\end{aligned}
$$

With every control $u \in L^{2}(0, T ; Y)$, we consider the following distributional cost function expressed by

$$
J_{1}(u)=\int_{0}^{T}\left\|C x_{u}(t)-z_{d}(t)\right\|_{X}^{2} d t+\int_{0}^{T}(R u(t), u(t)) d t,
$$

where the operator $C$ is bounded from $H$ to another Hilbert space $X$ and $z_{d} \in L^{2}(0, T ; X)$. Finally we are given that $R$ is a self adjoint and positive definite:

$$
R \in \mathscr{L}(X), \quad(R u, u) \geq c\|u\|, \quad c>0 .
$$

Let $x_{u}(t)$ stand for solution of (1) associated with the control $u \in L^{2}(0, T ; Y)$. Let $\mathcal{U}_{\mathrm{ad}}$ be a closed convex subset of $L^{2}(0, T ; Y)$.

Theorem 13. Let the assumptions in Theorem 12 be satisfied and let the operators $C$ and $N$ satisfy the conditions mentioned above. Then there exists an element $u \in \mathscr{U}_{\mathrm{ad}}$ such that

$$
J_{1}(u)=\inf _{v \in \mathscr{U}_{\mathrm{ad}}} J_{1}(v) .
$$

Furthermore, the following inequality holds:

$$
\int_{0}^{T}\left(\Lambda_{Y}^{-1} B^{*} p_{u}(t)+R u(t),(v-u)(t)\right) d t \geq 0, \quad \forall v \in \mathscr{U}_{\mathrm{ad}}
$$

holds, where $\Lambda_{Y}$ is the canonical isomorphism $Y$ onto $Y^{*}$ and $p_{u}$ satisfies the following equation:

$$
\begin{aligned}
& p_{u}^{\prime}(t)-A^{*} p_{u}(t)-D(\partial \phi)^{0}(x)^{*} p_{u}(t)+\partial_{2} f(t, x)^{*} p_{u}(t) \\
& \quad=-C^{*} \Lambda_{X}\left(C x_{u}(t)-z_{d}(t)\right), \quad \text { for } 0<t \leq T, \\
& P_{u}(T)=0 .
\end{aligned}
$$

Proof. Let $x(t)=x_{0}(t)$ be a solution of (1) associated with the control 0 . Then it holds that

$$
\begin{aligned}
J_{1}(v)= & \int_{0}^{T}\left\|C x_{v}(t)-z_{d}(t)\right\|_{X}^{2} d t+\int_{0}^{T}(R v(t), v(t)) d t \\
= & \int_{0}^{T}\left\|C\left(x_{v}(t)-x(t)\right)+C x(t)-z_{d}(t)\right\|_{X}^{2} d t \\
& +\int_{0}^{T}(R v(t), v(t)) d t \\
= & \pi(v, v)-2 L(v)+\int_{0}^{T}\left\|z_{d}(t)-C x(t)\right\|_{X}^{2} d t,
\end{aligned}
$$

where

$$
\begin{aligned}
\pi(u, v)= & \int_{0}^{T}\left(C\left(x_{u}(t)-x(t)\right), C\left(x_{v}(t)-x(t)\right)\right)_{X} d t \\
& \quad+\int_{0}^{T}(R u(t), v(t)) d t \\
L(v)= & \int_{0}^{T}\left(z_{d}(t)-C x(t), C\left(x_{v}(t)-x(t)\right)\right)_{X} d t .
\end{aligned}
$$

The form $\pi(u, v)$ is a continuous form in $L^{2}(0, T ; Y) \times$ $L^{2}(0, T ; Y)$ and from assumption of the positive definite of the operator $R$, we have

$$
\pi(v, v) \geq c\|v\|^{2}, \quad v \in L^{2}(0, T ; Y)
$$

If $u$ is an optimal control, similarly for (97), (84) is equivalent to

$$
\begin{gathered}
\int_{0}^{T}\left(C^{*} \Lambda_{X}\left(C x_{u}(t)-z_{d}(t)\right), y(t)\right) d t \\
+\int_{0}^{T}(R u(t),(v-u)(t)) d t \geq 0
\end{gathered}
$$

Now we formulate the adjoint system to describe the optimal condition:

$$
\begin{aligned}
p_{u}^{\prime}(t) & -A^{*} p_{u}(t)-D \partial \phi_{\epsilon}(x)^{*} p_{u}(t)+\partial_{2} f(t, x)^{*} p_{u}(t) \\
& =-\left(C^{*} \Lambda_{X} C x_{u}(t)-z_{d}(t)\right), \quad \text { for } 0<t \leq T, \\
P_{u}(T) & =0 .
\end{aligned}
$$

Taking into account the regularity result of Proposition 3 and the observation conditions, we can assert that (112) admits a unique weak solution $p_{u}$ reversing the direction of time $t \rightarrow T-t$ by referring to the well-posedness result of Dautray and Lions [19, pages 558-570]. 
We multiply both sides of (112) by $y(t)$ of (98) and integrate it over $[0, T]$. Then we have

$$
\begin{aligned}
\int_{0}^{T}( & \left.C^{*} \Lambda_{X}\left(C x_{u}(t)-z_{d}(t)\right), y(t)\right) d t \\
= & -\int_{0}^{T}\left(p_{u}^{\prime}(t), y(t)\right) d t+\int_{0}^{T}\left(A^{*} p_{u}(t), y(t)\right) d t \\
& +\int_{0}^{T}\left(D \partial \phi_{\epsilon}(x)^{*} p_{u}(t), y(t)\right) d t \\
& -\int_{0}^{T}\left(\partial_{2} f(t, x)^{*} p_{u}(t), y(t)\right) d t .
\end{aligned}
$$

By the initial value condition of $y$ and the terminal value condition of $p_{u}$, the left hand side of (113) yields

$$
\begin{aligned}
& -\left(p_{u}(T), y(T)\right)+\left(p_{u}(0), y(0)\right) \\
& \quad+\int_{0}^{T}\left(p_{u}(t), y^{\prime}(t)\right) d t+\int_{0}^{T}\left(p_{u}(t), A y(t)\right) d t \\
& \quad+\int_{0}^{T}\left(p_{u}(t), D \partial \phi_{\epsilon}(x) y(t)\right) d t \\
& \quad-\int_{0}^{T}\left(p_{u}(t), \partial_{2} f(t, x) y(t)\right) d t \\
& =\int_{0}^{T}\left(p_{u}(t), B(v-u)(t)\right) d t
\end{aligned}
$$

Let $u$ be the optimal control subject to (103). Then (111) is represented by

$$
\int_{\Omega}\left(p_{u}(t), B(v-u)(t)\right) d t+\int_{0}^{T}(R u(t),(v-u)(t)) d t \geq 0,
$$

which is rewritten by (106). Note that $C^{*} \in B\left(X^{*}, H\right)$ and for $\phi$ and $\psi$ in $H$ we have $\left(C^{*} \Lambda_{X} C \psi, \phi\right)=\langle C \psi, C \phi\rangle_{X}$, where duality pairing is also denoted by $(\cdot, \cdot)$.

Remark 14 . Identifying the antidual $X$ with $X$ we need not use the canonical isomorphism $\Lambda_{X}$. However, in case where $X \subset V^{*}$ this leads to difficulties since $H$ has already been identified with its dual.

\section{Acknowledgment}

This research was supported by Basic Science Research Program through the National research Foundation of Korea (NRF) funded by the Ministry of Education, Science and Technology (2012-0007560).

\section{References}

[1] V. Barbu, Analysis and Control of Nonlinear Infinite-Dimensional Systems, vol. 190, Academic Press, Boston, Mass, USA, 1993.

[2] G. Bertotti and I. Mayergoyz, The Science of Hysteresis, Academic Press, Elsevier, 2006.
[3] J.-M. Jeong and J.-Y. Park, "Nonlinear variational inequalities of semilinear parabolic type," Journal of Inequalities and Applications, vol. 6, no. 2, pp. 227-245, 2001.

[4] J.-L. Lions, "Quelques problèmes de la théorie des équations non linéaires d'évolution," in Problems in Non-Linear Analysis, pp. 189-342, Edizioni Cremonese, Rome, Italy, 1971.

[5] V. Barbu, Optimal Control of Variational Inequalities, vol. 100 of Research Notes in Mathematics, Pitman, Boston, Mass, USA, 1984.

[6] D. Kinderlehrer and G. Stampacchia, An Introduction to Variational Inequalities and Their Applications, vol. 88, Academic Press, New York, NY, USA, 1980.

[7] M. Shillor, Recent Advances in Contact Mechanics, Pergamon Press, 1998.

[8] J. F. Bonnans and D. Tiba, "Pontryagin's principle in the control of semilinear elliptic variational inequalities," Applied Mathematics and Optimization, vol. 23, no. 3, pp. 299-312, 1991.

[9] J.-L. Lions, Optimal Control of Systems Governed by Partial Differential Equations, Springer, New York, NY, USA, 1971.

[10] H. Triebel, Interpolation Theory, Function Spaces, Differential Operators, vol. 18 of North-Holland Mathematical Library, North-Holland, Amsterdam, The Netherlands, 1978.

[11] G. Di Blasio, K. Kunisch, and E. Sinestrari, " $L^{2}$-regularity for parabolic partial integro-differential equations with delay in the highest-order derivatives," Journal of Mathematical Analysis and Applications, vol. 102, no. 1, pp. 38-57, 1984.

[12] J. M. Jeong, Y. C. Kwun, and J. Y. Park, "Approximate controllability for semilinear retarded functional-differential equations," Journal of Dynamical and Control Systems, vol. 5, no. 3, pp. 329346, 1999.

[13] H. Tanabe, Equations of Evolution, vol. 6 of Monographs and Studies in Mathematics, Pitman, Boston, Mass, USA, 1979.

[14] H. Brézis, Opérateurs Maximaux Monotones et Semigroupes de Contractions dans un Espace de Hilbert, North Holland, 1973.

[15] J. Hwang and S. Nakagiri, "Optimal control problems for the equation of motion of membrane with strong viscosity," Journal of Mathematical Analysis and Applications, vol. 321, no. 1, pp. 327-342, 2006.

[16] J. Hwang and S. Nakagiri, "Optimal control problems for Kirchhoff type equation with a damping term," Nonlinear Analysis: Theory, Methods \& Applications, vol. 72, no. 3-4, pp. 1621-1631, 2010.

[17] J.-P. Aubin, "Un théorème de compacité," Comptes Rendus de l’Académie des Sciences, vol. 256, pp. 5042-5044, 1963.

[18] V. Barbu, Nonlinear Semigroups and Differential Equations in Banach Spaces, Nordhoff, Leiden, The Netherlands, 1976.

[19] R. Dautray and J. L. Lions, "Mathematical analysis and numerical methods for science and technology," in Evolution Problems, vol. 5, Springer, 1992. 


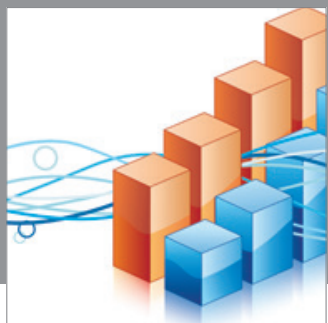

Advances in

Operations Research

mansans



The Scientific World Journal
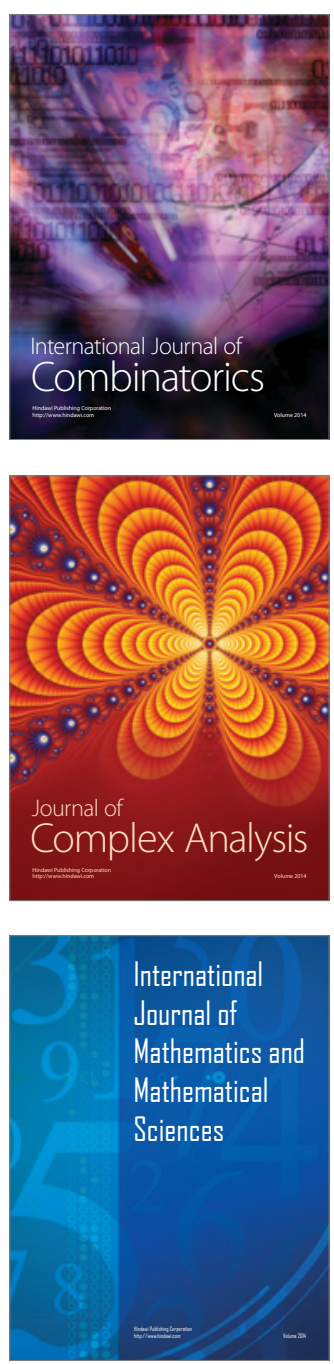
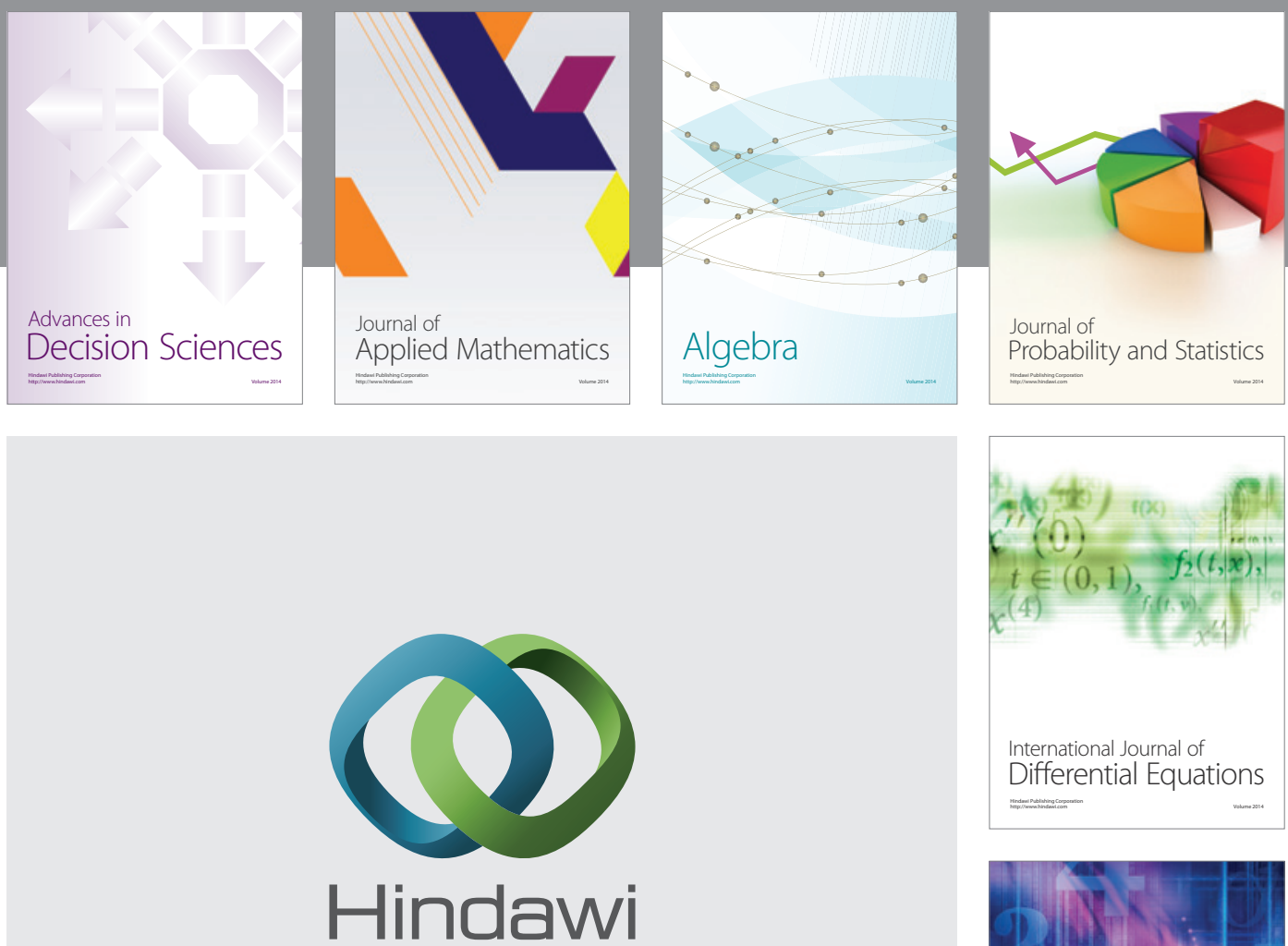

Submit your manuscripts at http://www.hindawi.com


Journal of

Function Spaces

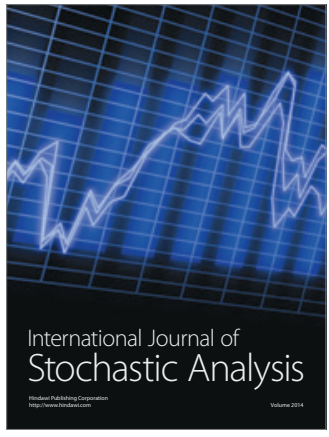


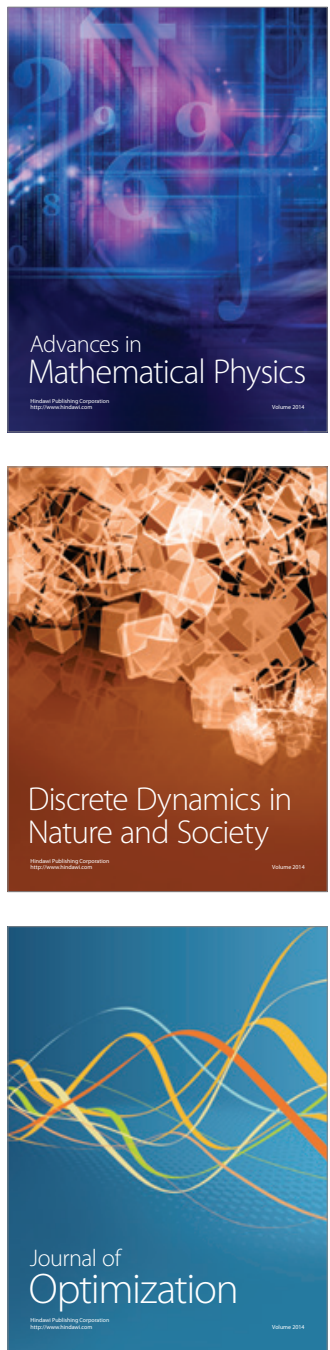